\title{
Ordinal Completeness of Bimodal Provability Logic GLB
}

\author{
Dedicated to Leo Esakia on the occasion of his 75-th birthday
}

\author{
Lev Beklemishev*
}

Steklov Mathematical Institute, Gubkina 8, 119991, Moscow, Russia

\begin{abstract}
Bimodal provability logic GLB, introduced by G. Japaridze, currently plays an important role in the applications of provability logic to proof-theoretic analysis. Its topological semantics interprets diamond modalities as derived set operators on a scattered bitopological space. We study the question of completeness of this logic w.r.t. the most natural space of this kind, that is, w.r.t. an ordinal $\alpha$ equipped with the interval topology and with the so-called club topology. We show that, assuming the axiom of constructibility, GLB is complete for any $\alpha \geq \aleph_{\omega}$. On the other hand, from the results of A. Blass it follows that, assuming the consistency of "there is a Mahlo cardinal," it is consistent with ZFC that GLB is incomplete w.r.t. any such space. Thus, the question of completeness of GLB w.r.t. natural ordinal spaces turns out to be independent of ZFC.
\end{abstract}

\section{Introduction}

This paper links together two topics in the study of provability logic both of which originated in Georgia. The first one is the interpretation of modal $\diamond$ of the standard provability logic GL in topological terms as the derived set operator on a scattered topological space. The idea of treating topological derivative as a modality goes back to McKinsey and Tarski [22], however it was Leo Esakia who linked scattered spaces with the axioms of the logic of provability (see $[15,16])$. In particular, Esakia proved that GL was complete under this interpretation w.r.t. the class of all scattered spaces. Merab Abashidze [1] and later independently Andreas Blass [11] established the completeness of $\mathbf{G L}$ w.r.t. some natural individual scattered spaces. Thus, Abashdze and Blass showed the completeness of GL w.r.t. the standard interval topology on any ordinal $\alpha \geq \omega^{\omega}$. In addition, by an interesting combinatorial construction, Blass also showed GL to be complete w.r.t. another natural topology on an ordinal $\alpha$, the so-called club topology, provided $\alpha \geq \aleph_{\omega}$. However, this latter result could only be proved assuming the set-theoretic axiom of constructibility (or Jensen's square principle). In fact, assuming the consistency of "there is a Mahlo cardinal," Blass also showed that

\footnotetext{
* Research supported by the Russian Foundation for Basic Research (RFBR) and Russian Presidential Council for Support of Leading Scientific Schools.
} 
it is consistent with the axioms of ZFC that GL is incomplete w.r.t. the club topology on any ordinal. Both the club topology and Blass's theorem will play a prominent role in this paper.

The second topic that emerged in Georgia is the extension of the language of GL by an infinite sequence of new diamond modalities $\langle 0\rangle,\langle 1\rangle,\langle 2\rangle, \ldots$ corresponding to $n$-consistency predicates in first-order arithmetic. (Under this interpretation, $\langle 0\rangle$ means the same as the usual $\diamond$, i.e., consistency over a given theory.) This leads to a much more expressive polymodal provability logic GLP, which was first formulated and proved arithmetically complete by Giorgi Japaridze [19]. His study has later been simplified and extended by Konstantin Ignatiev [18] and George Boolos [13,12]. More recently, GLP has found interesting applications in proof-theoretic analysis of arithmetic $[3,4,2,5]$ which stimulated some further interest in the study of modal-logical properties of GLP $[14,9,17,6,7]$.

Unlike $\mathbf{G L}$, which is complete w.r.t. its Kripke semantics, that is, w.r.t. the class of finite irreflexive trees, it is well-known that even the bimodal fragment of GLP, denoted GLB by Boolos, is not complete w.r.t. any class of Kripke frames. Therefore, the problem of finding a tractable complete semantics for this logic becomes more urgent. Topological semantics can be considered as a generalization of Kripke semantics, therefore it is natural to ask if there is a complete topological semantics for GLP.

Esakia-style topological models for GLP have been introduced in [8] under the name GLP-spaces. These are polytopological spaces of the form $\left(X ; \tau_{0}, \tau_{1}, \ldots\right)$, where modality $\langle n\rangle$ is interpreted as a derived set operator $d_{n}$ on $X$ w.r.t. topology $\tau_{n}$. To satisfy all the axioms of GLP we must require, for each $n$, that

- $\tau_{n}$ is a scattered topology on $X$;

$-\tau_{n} \subseteq \tau_{n+1}$

- for all $A \subseteq X, d_{n}(A)$ is $\tau_{n+1}$-open.

$\boldsymbol{G} \boldsymbol{L} \boldsymbol{B}$-spaces are structures $\left(X ; \tau_{0}, \tau_{1}\right)$ of the same kind with only the first two topologies present. The simplest nontrivial example of a GLB-space is an ordinal space of the form $\left(\alpha ; \tau_{0}, \tau_{1}\right)$ where $\tau_{0}$ is the interval topology on an ordinal $\alpha$, and $\tau_{1}$ is the above mentioned club topology. In fact, $\tau_{1}$ happens to be the coarsest topology such that $\left(\alpha ; \tau_{0}, \tau_{1}\right)$ is a GLB-space, which can be considered as a definition of $\tau_{1}$ (see [8]). Thus, the general notion of GLB-space links the two prominent ordinal topologies: the interval topology and the club topology. ${ }^{1}$ We call such spaces natural ordinal $\boldsymbol{G} \boldsymbol{L} \boldsymbol{B}$-spaces.

In [8], it was shown that GLB is complete w.r.t. the class of all GLBspaces, thus, a bimodal analogue of Esakia theorem was established. However,

\footnotetext{
${ }^{1}$ Blass treated the club topology in terms of its (punctured) neighborhood filters - the so-called club filters. His interest in this semantics was motivated by the importance of the concept of club filter in set theory. In contrast, in our situation the club topology is more or less forced on us by the general concept of GLB-space, that is, by the axioms of GLB. The fact that we come in this way to an important set-theoretic notion additionally speaks for the naturality of the concept of GLBspace.
} 
two further questions were left open. Firstly, the question whether this result can be extended to the full language of GLP. Secondly, the question whether GLB is complete w.r.t. any natural (ordinal) GLB-space, that is, if an analog of Abashidze-Blass theorem holds for GLB. (A similar but more difficult question also makes sense for the full GLP.)

In this paper we answer the second question for the case of GLB in essentially the same sense as Blass. Namely, under the assumption of the axiom of constructibility, we show that GLB is complete w.r.t. any natural ordinal GLBspace of the form $\left(\alpha ; \tau_{0}, \tau_{1}\right)$ where $\alpha \geq \aleph_{\omega}$. This result can be considered as an extension of both topological completeness theorems of Abashidze and Blass. In fact, the proof of our theorem relies on Blass's construction in an essential way.

The question of general topological completeness of GLP has recently been affirmatively answered jointly by David Gabelaia and the author of this paper. This result is based on some additional techniques and will be published separately.

\section{Preliminaries and statement of main result}

\subsection{Axioms of GLB}

We consider the language of propositional logic enriched by two modalities [0] and [1]. Dual modalities are denoted $\langle 0\rangle$ and $\langle 1\rangle$ and treated as abbreviations for $\neg[0] \neg$ and $\neg[1] \neg$ respectively. The system GLB is given by the following axiom schemata and inference rules:

\section{Axioms:}

(i) Boolean tautologies;

(ii) $[n](\varphi \rightarrow \psi) \rightarrow([n] \varphi \rightarrow[n] \psi)$ (for $n=0,1)$;

(iii) $[n]([n] \varphi \rightarrow \varphi) \rightarrow[n] \varphi($ for $n=0,1)$;

(iv) $[0] \varphi \rightarrow[1] \varphi$

Rules:

(v) $\langle 0\rangle \varphi \rightarrow[1]\langle 0\rangle \varphi$.

(i) $\vdash \varphi, \vdash \varphi \rightarrow \psi \Rightarrow \vdash \psi$ (modus ponens);

(ii) $\vdash \varphi \Rightarrow \vdash[n] \varphi$, for $n=0,1$ (necessitation).

\subsection{Topological semantics}

Let $\left(X ; \theta_{0}, \theta_{1}\right)$ be a bitopological space. For $n=0,1$ let $d_{n}(A)$ denote the set of limit points of $A \subseteq X$ w.r.t. $\theta_{n}$, that is, $x \in d_{n}(A)$ iff $A$ intersects any neighborhood $U$ of $x$ at a point $y \neq x$. Thus, $d_{0}$ and $d_{1}$ denote the derived set operators associated with the topologies $\theta_{0}$ and $\theta_{1}$.

When $X$ is fixed and $A \subseteq X$ we write $-A$ for $X-A$. We also denote $\tilde{d}_{n}(A)=-d_{n}(-A)$. The operator $\tilde{d}_{n}$ will interpret $[n]$, whereas $d_{n}$ will interpret $\langle n\rangle$.

A valuation on $X$ is a map $v:$ Form $\rightarrow \mathcal{P}(X)$ of propositional formulas to subsets of $X$ such that 
$-v(\varphi \wedge \psi)=v(\varphi) \cap v(\psi), v(\neg \varphi)=-v(\varphi)$, etc.;

$-v(\langle n\rangle \varphi)=d_{n}(v(\varphi)), v([n] \varphi)=\tilde{d}_{n}(v(\varphi))$, for $n=0,1$.

A formula $\varphi$ is valid in $X$ if $v(\varphi)=X$, for any valuation $v$ on $X$. This fact will be denoted $\left(X ; \theta_{0}, \theta_{1}\right) \vDash \varphi$. The logic of $\left(X ; \theta_{0}, \theta_{1}\right)$ is the set of all formulas valid in $X$ :

$$
L\left(X ; \theta_{0}, \theta_{1}\right)=\left\{\varphi:\left(X ; \theta_{0}, \theta_{1}\right) \vDash \varphi\right\} .
$$

\subsection{Ordinal GLB-spaces}

We consider bitopological spaces of the form $\left(\kappa ; \tau_{0}, \tau_{1}\right)$, where $\kappa$ is an ordinal, $\tau_{0}$ is the interval topology on $\kappa$, and $\tau_{1}$ is the club topology. As usual, with the topologies $\tau_{0}$ and $\tau_{1}$ we associate derived set operators $d_{0}$ and $d_{1}$.

Recall that $\tau_{0}$ is generated by $\{0\}$ and intervals of the form $(\alpha, \beta]$, for all $\alpha<\beta<\kappa$. Notice that $d_{0}(A)$ is often denoted $A^{\prime}$. We have: $\alpha \in d_{0}(A)$ iff ( $\alpha$ is a limit ordinal and $A \cap \alpha$ is unbounded in $\alpha$ ). Topology $\tau_{0}$ is scattered, that is, every non-empty subspace $A \subseteq \kappa$ has an isolated point; as such a point one can take the minimum of $A$.

By definition, the club topology $\tau_{1}$ on $\kappa$ is generated by $\tau_{0}$ and all sets of the form $d_{0}(A)$, where $A \subseteq \kappa$. Since $\tau_{0} \subseteq \tau_{1}$ we observe that $\tau_{1}$ is also scattered.

A subset $C \subseteq \alpha$ is called a club in $\alpha$, if $C$ is $\tau_{0}$-closed in the relative topology of $\alpha$ and unbounded in $\alpha$. Notice that if $\alpha \in d_{0}(A)$ then $d_{0}(A) \cap \alpha$ is a club in $\alpha$. If $\alpha$ is a limit ordinal of uncountable cofinality $\operatorname{cf}(\alpha)$, the intersection of finitely many clubs in $\alpha$ is again a club. It is easy to infer from this property that the limit points of $\tau_{1}$ (that is, points of the set $d_{1}(\kappa)$ ) are exactly the ordinals of uncountable cofinality below $\kappa$.

Similarly, we can characterize $\tau_{1}$ in terms of neighborhoods: $A$ is a $\tau_{1}$ neighborhood of $\alpha$ iff $\alpha \in A$ and either $\operatorname{cf}(\alpha) \leq \omega$ or $A$ contains a club in $\alpha$. As a consequence, we obtain the following characterization of derivative $d_{1}$ : $\alpha \in d_{1}(A)$ iff $(\operatorname{cf}(\alpha)>\omega$ and $A \cap \alpha$ intersects every club $C$ in $\alpha)$. A subset $A \subseteq \alpha$ is called stationary in $\alpha$ if $A$ intersects every club in $\alpha$. Thus, $\alpha \in d_{1}(A)$ iff $(\operatorname{cf}(\alpha)>\omega$ and $A \cap \alpha$ is stationary in $\alpha)$. In set theory, one usually calls $d_{1}$ Mahlo operation (see [20]).

Let $D_{\kappa}^{0}=\kappa$ and $D_{\kappa}^{n}$ denote $\left\{\alpha<\kappa: \operatorname{cf}(\alpha) \geq \aleph_{n}\right\}$ for $n>0$. The following simple lemma is well-known and will be useful below.

Lemma 1. For any $n, D_{\kappa}^{n}=d_{1}^{n}(\kappa)$.

Proof. By induction, it is sufficient to show that

$$
d_{1}\left(D_{\kappa}^{n}\right)=D_{\kappa}^{n+1} .
$$

Suppose $\lambda \in D_{\kappa}^{n+1}$ and $C$ is a club in $\lambda$. Let $\alpha$ be the $\aleph_{n}$-th element of $C$. Since $\aleph_{n}$ is regular, $\operatorname{cf}(\alpha) \geq \aleph_{n}$, hence $\alpha \in D_{\kappa}^{n}$. Therefore, $d_{1}\left(D_{\kappa}^{n}\right) \supseteq D_{\kappa}^{n+1}$.

Conversely, consider an ordinal $\lambda \notin D_{\kappa}^{n+1}$. If $\operatorname{cf}(\lambda) \leq \omega$, clearly $\lambda$ is not a limit point of $D_{\kappa}^{n}$. Otherwise, $\operatorname{cf}(\lambda)<\aleph_{n+1}$, hence there is a club $C$ in $\lambda$ whose order type ot $(C)$ satisfies $\aleph_{1} \leq$ ot $(C) \leq \aleph_{n}$. Note that $d_{0}(C) \cap \lambda$ is a club in $\lambda$ and every $\alpha \in d_{0}(C) \cap \lambda$ satisfies $\operatorname{cf}(\alpha)<\operatorname{ot}(C)$, hence $\operatorname{cf}(\alpha)<\aleph_{n}$. Therefore, $D_{\kappa}^{n} \cap d_{0}(C)$ is empty and $\lambda$ is not a limit point of $D_{\kappa}^{n}$. 


\subsection{Main theorem}

We are interested in characterizing the logic $L\left(\kappa ; \tau_{0}, \tau_{1}\right)$ for natural ordinal GLBspaces $\left(\kappa ; \tau_{0}, \tau_{1}\right)$. Since $\tau_{0}$ is scattered, $\tau_{0} \subseteq \tau_{1}$ and every set of the form $d_{0}(A)$ is $\tau_{1}$-open, it is easy to see that $\left(\kappa ; \tau_{0}, \tau_{1}\right)$ is a GLB-space, in particular, all theorems of GLB are valid in $\left(\kappa ; \tau_{0}, \tau_{1}\right)$.

We show that under some standard set-theoretic assumptions, for ordinals $\kappa \geq \aleph_{\omega}$, the space $\left(\kappa ; \tau_{0}, \tau_{1}\right)$ is, in fact, complete for GLB. Since the completeness of GLB implies the completeness of GL w.r.t. the club topology on $\kappa$, it follows from the results of Blass [11] that some set-theoretic assumptions beyond ZFC are necessary for the completeness proof.

For any infinite cardinal $\kappa$, Jensen's Principle $\square_{\kappa}$ is the assertion that there exists a sequence of sets $C_{\alpha}$, for limit ordinals $\alpha<\kappa^{+}$, with the following properties:

(i) $C_{\alpha}$ is a club in $\alpha$;

(ii) If $\operatorname{cf}(\alpha)<\kappa$ then $\left|C_{\alpha}\right|<\kappa$;

(iii) If $\beta \in d_{0}\left(C_{\alpha}\right)$ then $C_{\beta}=\beta \cap C_{\alpha}$.

It is a well-known result of Jensen that the axiom of constructibility $V=L$ implies $\square_{\kappa}$, for all $\kappa$. (For $\kappa=\aleph_{0}$ the assertion $\square_{\kappa}$ trivializes and is provable in ZFC. See Jech [20] for the details on any specific set-theoretic notions and facts mentioned in this paper.)

Under the assumption of $\square_{\aleph_{n}}$, for every $n<\omega$, we prove the following completeness theorem.

Theorem $1\left(\square_{\aleph_{n}}\right.$, for $\left.n<\omega\right)$. For any $\kappa \geq \aleph_{\omega}, L\left(\kappa ; \tau_{0}, \tau_{1}\right)=\boldsymbol{G L} \boldsymbol{B}$.

A proof of this theorem will be obtained by combining several ingredients. We will use a reduction of GLB to a subsystem $\mathbf{J}$ isolated in [6], which is complete w.r.t. a nice class of finite Kripke frames called treelike $J$-frames. Then we will state a general Embedding lemma which allows to 'embed' such treelike $J$-frames into ordinal spaces. Thus, given a formula $\varphi$ such that GLB $\nvdash \varphi$ we will be able to produce a valuation on $\left(\left[1, \aleph_{n}\right] ; \tau_{0}, \tau_{1}\right)$ falsifying $\varphi$. Finally, all such valuations will be merged into a single one on the space $\left(\aleph_{\omega} ; \tau_{0}, \tau_{1}\right)$.

In Section 4 we state the Embedding lemma and infer from it Theorem 1. A proof of the Embedding lemma is postponed until Section 5. In Section 3 we formulate a lemma summarizing the key properties of Blass's construction necessary for the statement and the proof of the Embedding lemma.

\section{Blass's construction}

The proof of Theorem 1 will heavily rely on the previous results of Andreas Blass. The following lemma can be extracted from his paper [11].

Lemma $2\left(\square_{\aleph_{n}}\right.$, for $\left.n<\omega\right)$. For any finite tree $(T, \prec)$ of height $n$ there is a map $S$ associating with every $x \in T$ a nonempty subset $S_{x} \subseteq\left[1, \aleph_{n}\right]$ with the following properties: 
(i) $\left\{S_{x}: x \in T\right\}$ is a partition of $\left[1, \aleph_{n}\right]$;

(ii) $S_{r}=\left\{\aleph_{n}\right\}$, if $r \in T$ is the root of $T$;

(iii) If $x \prec y$ then $S_{x} \subseteq d_{1}\left(S_{y}\right)$; in other words, if $x \prec y$ and $\lambda \in S_{x}$ then $\operatorname{cf}(\lambda)>\omega$ and $S_{y} \cap \lambda$ is stationary in $\lambda$;

(iv) $S_{x} \subseteq \tilde{d}_{1}\left(\bigcup_{y \succ x} S_{y}\right)$; in other words, if $\lambda \in S_{x}$ and $\operatorname{cf}(\lambda)>\omega$, then $\bigcup_{y \succ x} S_{y}$ contains a club in $\lambda$.

Rather than being literally stated in Blass's paper, this lemma is obtained by some adaptation of the construction in his proof. Before discussing the relationship in more detail a few general remarks are in order.

Firstly, we notice that the conditions of this lemma are quite similar to those of the Solovay construction in provability logic. Therefore, in a similar manner, it is easy to infer from it the completeness of $\mathbf{G L}$ w.r.t. the topological space $\left(\aleph_{\omega}, \tau_{1}\right)$. This is one of the two main results of Blass's paper; the inference of completeness from an analogue of this lemma is essentially the content of his Theorem 3.

Secondly, with the map $S: x \longmapsto S_{x}$ we can associate a surjective function $f:\left[1, \aleph_{n}\right] \rightarrow T$ mapping every point of $S_{x}$ to $x$. Let $(T, \prec)$ be equipped with the Alexandroff topology whose open sets are exactly the upwards closed ones w.r.t. the ordering $\prec$. Conditions (iii) and (iv) are then equivalent to $f$ being continuous, open and pointwise discrete (the latter means that $f^{-1}\{a\}$ is discrete, for each $a \in T$ ). Such functions $f$ have been introduced in [10] under the name $d$-maps. They play for our kind of topological semantics a role similar to the one of p-morphism for Kripke frames. Thus, Lemma 2 can be restated as follows.

Corollary $1\left(\square_{\aleph_{n}}\right.$, for $\left.n<\omega\right)$. For every finite tree $(T, \prec)$ of depth $n$ there is a surjective d-map $f:\left[1, \aleph_{n}\right] \rightarrow T$.

If $f: X \rightarrow Y$ is a $d$-map and $v$ is a valuation on $Y$, we can associate with it a valuation $v^{\prime}$ on $X$ by defining, for each variable $p$,

$$
v^{\prime}(p):=\{x \in X: f(x) \in v(p)\} .
$$

Then it is easy to see that $v^{\prime}(\varphi)=f^{-1}(v(\varphi))$, for each formula $\varphi$. Hence, the logic of $X$ is contained in the logic of $Y$. This is another way of looking at the inference of the completeness of GL from Lemma $2 .{ }^{2}$

Thus, for the applications of Lemma 2 only Conditions (i), (iii) and (iv) are substantial. Condition (ii) plays a purely technical role in Blass's paper to make the inductive construction of the $d$-map work. In fact, from the next lemma we can conclude that Condition (ii) actually follows from the other three conditions.

Let $\operatorname{dp}(x)$ denote the depth of a node $x \in T$ w.r.t. the ordering $\prec$. The height of $T$ is the depth of its root.

\footnotetext{
${ }^{2}$ We can also look at the same situation algebraically. A surjective $d$-map $f: X \rightarrow Y$ induces a dual map $f^{*}: \mathcal{P}(Y) \rightarrow \mathcal{P}(X)$ defined by $f^{*}(A):=f^{-1}(A)$, which happens to be a homomorphic embedding of the modal algebra $\left(\mathcal{P}(Y), d_{Y}\right)$ into $\left(\mathcal{P}(X), d_{X}\right)$. In particular, this justifies the name 'Embedding lemma' for the results of this kind.
} 
Lemma 3. Let $f:\left[1, \aleph_{n}\right] \rightarrow(T, \prec)$ be a surjective $d$-map. Then, for any $\alpha \in$ $\left[1, \aleph_{n}\right]$ and $k \leq n, \operatorname{cf}(\alpha)=\omega_{k}$ iff $\operatorname{dp}(f(\alpha))=k$.

Proof. Let $d$ denote the derivative operation w.r.t. the Alexandroff topology of the tree $(T, \prec)$. Thus,

$$
d(A)=\{x \in T: \exists y \in A x \prec y\} .
$$

We obviously have, by the definition of depth:

$$
\operatorname{dp}(x) \geq n \Longleftrightarrow x \in d^{n}(T) .
$$

Since $f$ is a surjective $d$-map, it satisfies $f^{-1}(d A)=d_{1}\left(f^{-1}(A)\right)$, for each $A \subseteq T$. Hence, $f^{-1}\left(d^{k} T\right)=d_{1}^{k}\left[1, \aleph_{n}\right]$. In turn, from Lemma 1 for $\alpha \in\left[1, \aleph_{n}\right]$, we conclude that $\alpha \in d_{1}^{k}\left[1, \aleph_{n}\right]$ iff $\operatorname{cf}(\alpha) \geq \aleph_{k}$, whence the claim.

Corollary 2. Condition (ii) follows from (i), (iii) and (iv).

Proof. The root is the only point of $T$ of maximal depth.

Recall that the ordinal function $f(\alpha)=\omega \alpha$ is monotone and continuous w.r.t. $\tau_{0}$. It enumerates 0 and all limit ordinals in the increasing order, so it maps $X=\left[1, \aleph_{n}\right]$ onto $Y=\left[1, \aleph_{n}\right] \cap$ Lim. We consider $Y \subseteq X$ equipped with the topologies inherited from $X$. The following lemma is straightforward.

Lemma 4. $f: X \rightarrow Y$ is a homeomorphism w.r.t. both $\tau_{0}$ and $\tau_{1}$.

Proof. $f$ is obviously bijective and continuous w.r.t. $\tau_{0}$. Since $f$ is monotone, $f(\alpha, \beta]=(f(\alpha), f(\beta)] \cap Y$. Hence, $f(\alpha, \beta]$ is open in $Y$. Therefore, $f$ is open and hence a homeomorphism w.r.t. $\tau_{0}$.

Since $f$ is a homeomorphism and an order isomorphism, $C$ is a club in $\alpha$ if and only if $f(C)$ is a club in $f(\alpha)$, for any $C \subseteq X$. We show that $f$ is continuous and open w.r.t. $\tau_{1}$.

Suppose $U$ is open in $Y$ and $f(\alpha) \in U$. We show that there is a neighborhood $V$ of $\alpha$ such that $f(V) \subseteq U$. We consider two cases. If $\operatorname{cf}(f(\alpha))=\omega$, then $\operatorname{cf}(\alpha)=\omega$, hence $\{\alpha\}$ is open and $f\{\alpha\} \subseteq U$.

If $\operatorname{cf}(f(\alpha))>\omega$, then $U=U_{0} \cap Y$ where $U_{0}$ contains a club $C$ in $f(\alpha)$. Obviously, $C^{\prime}=d_{0}(C) \subseteq C$ is a club in $f(\alpha)$ contained in $Y$. Then $f^{-1}\left(C^{\prime}\right)$ is a club in $\alpha$, hence $V=\{\alpha\} \cup f^{-1}\left(C^{\prime}\right)$ is a neighborhood of $\alpha$ in $X$ and $f(V) \subseteq U$.

Suppose now $U$ is a neighborhood of $\alpha \in X$. If $\operatorname{cf}(\alpha)=\omega, \alpha$ is isolated in $X$ and $f(\alpha)$ is isolated in $Y$, as required. If $\operatorname{cf}(\alpha)>\omega$ we find a club $C \subseteq U$ in $\alpha$. Then $f(C)$ is a club in $f(\alpha)$, hence $f(U)$ contains a neighborhood of $f(\alpha)$ (both in $X$ and in $Y$ ).

Remark 1. In view of the previous lemma, Lemma 2 is equivalent to the one where Condition (i) is replaced by the requirement that $\left\{S_{x}: x \in T\right\}$ be a partition of the set $\left[1, \aleph_{n}\right] \cap$ Lim. In fact, Blass's original construction is formulated in this way. In our proof we will need both versions of Lemma 2. 
Remark 2. Another formal difference between Lemma 2 and [11] is that, rather than constructing a $d$-map from $\left[1, \aleph_{n}\right] \cap$ Lim to an arbitrary finite tree of depth $n$, Blass uses some explicit universal tree $K_{n}$ of depth $n$ and constructs a $d$ map from $\left[1, \aleph_{n}\right] \cap$ Lim to $K_{n}$. Universality here simply means that there is a surjective $d$-map from $K_{n}$ to any finite tree $T$ of depth $n$. Trees $K_{n}$ are infinitely branching and somewhat cumbersome to handle, and from the point of view of the proof of Theorem 1 nothing is gained by working with $K_{n}$, so we prefer to deal with the somewhat weaker statement of Lemma 2.

Remark 3. Since the spaces $[1, \kappa]$ and $[0, \kappa]$ are obviously isomorphic for infinite ordinals $\kappa$, the reader may wonder why we prefer to begin with 1 in the statement of Lemma 2. The answer is that this innocent choice greatly simplifies some formulas in the proof of the Embedding lemma.

\section{Embedding lemma for $J$-frames}

\section{$4.1 J$-frames}

Similarly to [8], our proof of topological completeness will make use of a subsystem of GLB introduced in [6] and denoted $\mathbf{J}$. This logic is defined by weakening axiom (iv) of GLB to the following axioms (vi) and (vii), both of which are theorems of GLB:

(vi) $[0] \varphi \rightarrow[1][0] \varphi$;

(vii) $[0] \varphi \rightarrow[0][1] \varphi$.

$\mathbf{J}$ is the logic of a simple class of frames, which is established by standard methods [6, Theorem 1].

Lemma 5. J is sound and complete with respect to the class of (finite) frames $\left(W ; R_{1}, R_{2}\right)$ such that, for all $x, y, z \in W$,

1. $R_{0}$ and $R_{1}$ are transitive and dually well-founded binary relations;

2. If $x R_{1} y$, then $x R_{0} z$ iff $y R_{0} z$;

3. $x R_{0} y$ and $y R_{1} z$ imply $x R_{0} z$.

If we let $\overline{R_{1}}$ denote the reflexive, symmetric, transitive closure of $R_{1}$, then we call each $\overline{R_{1}}$ equivalence class a 1-sheet. By 2., all points in a 1 -sheet are $R_{0}$ incomparable. But $R_{0}$ defines a natural ordering on the set of 1-sheets: if $\alpha$ and $\beta$ are 1-sheets, then $\alpha R_{0} \beta$, iff $\exists x \in \alpha, \exists y \in \beta, x R_{0} y$. By standard techniques, one can improve on Lemma 5 to show that $\mathbf{J}$ is complete for such frames, in which each 1 -sheet is a tree under $R_{1}$, and if $\alpha R_{0} \beta$ then $x R_{0} y$ for all $x \in \alpha, y \in \beta$ (see [6, Theorem 2 and Corollary 3.3]). Thus, models of $\mathbf{J}$ can be seen as $R_{0}$-orders (and even tree-like orders), in which the nodes are 1-sheets that are themselves $R_{1}$-trees. We call such frames tree-like J-frames. The $R_{1}$-height of such a frame is the maximum height of its 1-sheets. 
As shown in [6], GLB is reducible to $\mathbf{J}$ in the following sense. Let

$$
M(\varphi):=\bigwedge_{i<s}\left([0] \varphi_{i} \rightarrow[1] \varphi_{i}\right),
$$

where $[0] \varphi_{i}, i<s$, are all subformulas of $\varphi$ of the form [0] $\psi$. Also, let

$$
M^{+}(\varphi):=M(\varphi) \wedge[0] M(\varphi) \wedge[1] M(\varphi) .
$$

We have: GLB $\vdash \varphi$ iff $\mathbf{J} \vdash M^{+}(\varphi) \rightarrow \varphi$. In fact, below we only use the obvious part of this reduction, the implication from right to left.

\subsection{Embedding lemma}

For a binary relation $R$ on $X$ let $R(x)$ denote the set $\{y \in X: x R y\}$.

Lemma 6 (Embedding). Let $\mathcal{W}=\left(W ; R_{0}, R_{1}\right)$ be a finite tree-like $J$-frame with the root $r$ and $R_{1}$-height $n$. There is an ordinal $\kappa<\aleph_{n+1}$ and a map $S$ associating with every $x \in W$ a nonempty subset $S_{x} \subseteq[1, \kappa]$ with the following properties:

1. $\left\{S_{x}: x \in T\right\}$ is a partition of $[1, \kappa] ; S_{r}=\{\kappa\}$;

2. If $x R_{1} y$ then $S_{x} \subseteq d_{1}\left(S_{y}\right)$; in other words, if $x R_{1} y$ and $\lambda \in S_{x}$ then $\operatorname{cf}(\lambda)>\omega$ and $S_{y} \cap \lambda$ is stationary in $\lambda$;

3. If $x R_{0} y$ then $S_{x} \subseteq d_{0}\left(S_{y}\right)$; in other words, if $x R_{0} y$ and $\lambda \in S_{x}$ then $\lambda \in \operatorname{Lim}$ and $S_{y} \cap \lambda$ is unbounded in $\lambda$;

4. $S_{x} \subseteq \tilde{d}_{1}\left(\bigcup_{y \in R_{1}(x)} S_{y}\right)$; in other words, if $\lambda \in S_{x}$ and $\operatorname{cf}(\lambda)>\omega$, then $\bigcup_{y \in R_{1}(x)} S_{y}$ contains a club in $\lambda$;

5. $S_{x} \subseteq \tilde{d}_{0}\left(\bigcup_{y \in \widetilde{R}_{0}(x)} S_{y}\right)$; in other words, if $\lambda \in S_{x}$ and $\lambda \in \operatorname{Lim}$, then $\bigcup_{y \in \widetilde{R_{0}}(x)} S_{y}$ contains an end-segment of $\lambda$, where $\widetilde{R_{0}}(x):=R_{0}(x) \cup R_{1}\left(x_{1}\right)$ and $x_{1}$ denotes the root of the 1-sheet of $x$.

This lemma gives us a surjective function $f:[1, \kappa] \rightarrow W$ defined by $f^{-1}(x)=$ $S_{x}$. Such a function is a $d$-map w.r.t. $\tau_{1}$, open w.r.t. $\tau_{0}$, but is not, in general, $\tau_{0}$-continuous. Nonetheless, these conditions turn out to be sufficient for the completeness proof. We shall call such functions weak d-maps. A proof of this lemma will be given in Section 5.

Now we are ready to infer Theorem 1 from the Embedding lemma.

Proof. Suppose GLB $\nvdash \varphi$. Then, obviously, $\mathbf{J} \nvdash M^{+}(\varphi) \rightarrow \varphi$. Let $\mathcal{W}$ be a finite tree-like $J$-model with the root $r$ such that $\mathcal{W} \vDash M^{+}(\varphi)$ and $\mathcal{W} \nvdash \varphi$. Let $S$ be a map given by Lemma 6 . Define a valuation $v$ on $[1, \kappa]$ by $v(p):=\bigcup_{x \models p} S_{x}$, for each propositional variable $p$.

Lemma 7. For all subformulas $\theta$ of $\varphi$ we have:

$$
v(\theta)=\bigcup_{x \vDash \theta} S_{x} .
$$


Proof. Induction on the build-up of $\theta$. If $\theta$ is a variable, we refer to the definition of $v$. The cases $\theta=\psi_{1} \wedge \psi_{2}$ and $\theta=\neg \psi$ follow from the induction hypothesis and the fact that $S_{x}$ partition $[1, \kappa]$.

Let $\theta=[1] \psi$. If $\mathcal{W}, x \vDash \theta$ then $\forall y \in R_{1}(x) \mathcal{W}, y \vDash \psi$. Hence, by the induction hypothesis, $\forall y \in R_{1}(x) S_{y} \subseteq v(\psi)$, that is, $\bigcup_{y \in R_{1}(x)} S_{y} \subseteq v(\psi)$. By Lemma 6, Part 4,

$$
S_{x} \subseteq \tilde{d}_{1}\left(\bigcup_{y \in R_{1}(x)} S_{y}\right) \subseteq \tilde{d}_{1}(v(\psi)) .
$$

This shows $\bigcup_{x \models \theta} S_{x} \subseteq v(\theta)$.

If $\mathcal{W}, x \not \models \theta$ then $\exists y \in R_{1}(x) \mathcal{W}, y \not \models \psi$, and by the induction hypothesis $\exists y \in R_{1}(x) S_{y} \subseteq-v(\psi)$. By Lemma 6, Part 2,

$$
S_{x} \subseteq d_{1}\left(S_{y}\right) \subseteq d_{1}(-v(\psi))=-v(\theta) .
$$

This holds for all $x$ such that $\mathcal{W}, x \not \models \theta$, hence $v(\theta) \subseteq \bigcup_{x \models \theta} S_{x}$.

Let $\theta=[0] \psi$. If $\mathcal{W}, x \not \models \theta$ then $\exists y \in R_{0}(x) \mathcal{W}, y \not \models \psi$, and by the induction hypothesis $\exists y \in R_{0}(x) S_{y} \subseteq-v(\psi)$. By Lemma 6 , Part 3,

$$
S_{x} \subseteq d_{0}\left(S_{y}\right) \subseteq d_{0}(-v(\psi))=-v(\theta) .
$$

This holds for all $x$ such that $\mathcal{W}, x \not \models \theta$, hence $v(\theta) \subseteq \bigcup_{x \models \theta} S_{x}$.

If $\mathcal{W}, x \vDash \theta$ then $\forall y \in R_{0}(x) \mathcal{W}, y \vDash \psi$. Moreover, since $\mathcal{W}$ is a $J$-frame, we also have $\mathcal{W}, x_{1} \vDash[0] \psi$ (this is because $R_{0}(x)=R_{0}\left(x_{1}\right)$ in a $J$-frame). Since $\mathcal{W} \vDash M^{+}(\varphi)$ we have, in particular, $\mathcal{W}, x_{1} \vDash[0] \psi \rightarrow[1] \psi$ and hence $\mathcal{W}, x_{1} \vDash[1] \psi$. Therefore, $\forall y \in R_{1}\left(x_{1}\right) \mathcal{W}, y \vDash \psi$. So, we have $\forall y \in \widetilde{R_{0}}(x) \mathcal{W}, y \vDash \psi$ and by the induction hypothesis $\bigcup_{y \in \widetilde{R}_{0}(x)} S_{y} \subseteq v(\psi)$. By Lemma 6 , Part 5 ,

$$
S_{x} \subseteq \tilde{d}_{0}\left(\bigcup_{y \in \widetilde{R}_{0}(x)} S_{y}\right) \subseteq \tilde{d}_{0}(v(\psi))=v(\theta) .
$$

This holds for all $x$ such that $\mathcal{W}, x \vDash \theta$, hence $\bigcup_{x \vDash \theta} S_{x} \subseteq v(\theta)$.

Since $\mathcal{W} \not \models \varphi$, from the above lemma we conclude that $S_{x} \nsubseteq v(\varphi)$, hence $v(\varphi) \neq[1, \kappa]$. This shows that GLB is the intersection of logics of all GLBspaces $\left([1, \kappa], \tau_{0}, \tau_{1}\right)$, for $\kappa<\aleph_{\omega}$. To infer from this the stronger claim of the theorem, we need two additional general lemmas.

Let $\left(X_{i}\right)_{i \in I}$ be a family of GLB-spaces. The disjoint union $\bigsqcup_{i \in I} X_{i}$ of this family is defined in a natural way: $\tau_{n}$-open sets $A \subseteq \bigsqcup_{i \in I} X_{i}$ are those for which $A \cap X_{i}$ is $\tau_{n}$-open, for each $i \in I$. The following lemma is obvious.

Lemma 8. Suppose $f_{i}: X_{i} \rightarrow \mathcal{W}_{i}$ is a family of weak d-maps. Let $f: \bigsqcup_{i \in I} X_{i} \rightarrow$ $\bigsqcup_{i \in I} \mathcal{W}_{i}$ be such that $f \nmid X_{i}=f_{i}$, for each $i \in I$. Then $f$ is a weak $d$-map.

Lemma 9. Let $\left(\kappa_{\alpha}\right)_{\alpha<\lambda}$ be a family of non-zero ordinals and let $\kappa=\sum_{\alpha<\lambda} \kappa_{\alpha}$. Let $\tau_{0}$ and $\tau_{1}$ be the interval and the club topology on $\kappa$, respectively. Then $\boldsymbol{G L} \boldsymbol{B}$-space $\left([1, \kappa), \tau_{0}, \tau_{1}\right)$ is isomorphic to $\bigsqcup_{\alpha<\lambda}\left(\left[1, \kappa_{\alpha}\right], \tau_{0}, \tau_{1}\right)$. 
Proof. This is a consequence of two facts: 1$)$ An interval $(\alpha, \alpha+\beta]$ is isomorphic to $[1, \beta]$ both w.r.t. the order and the club topology; 2) The intervals $\left[1, \kappa_{0}\right]$ and $\left(\kappa_{\alpha}, \kappa_{\alpha+1}\right]$, for all $\alpha<\lambda$, are clopen w.r.t. both $\tau_{0}$ and $\tau_{1}$, and they partition $[1, \kappa)$.

Consider now some enumeration of all finite tree-like $J$-models $\left(\mathcal{W}_{i}\right)_{i<\omega}$. Let $f_{i}:\left[1, \kappa_{i}\right] \rightarrow \mathcal{W}_{i}$ be some surjective weak $d$-maps given by the Embedding Lemma. Applying the previous two lemmas, we obtain a surjective weak $d$-map $f:[1, \kappa) \rightarrow \bigsqcup_{i<\omega} \mathcal{W}_{i}$, where $\kappa:=\sum_{i<\omega} \kappa_{i}$. Hence, every formula falsified on some $\mathcal{W}_{i}$ is falsifiable on $[1, \kappa)$. Since $\kappa_{i}<\aleph_{\omega}$, for each $i$, we have $\kappa \leq \aleph_{\omega}$. From Lemma 3 and the fact that the sequence $\mathcal{W}_{i}$ contains $J$-frames of arbitrary finite $R_{1}$-depth one can infer that $\kappa \geq \aleph_{\omega}$, hence $\kappa=\aleph_{\omega}$. It follows that GLB is complete for $\aleph_{\omega}$ and therefore for any $\kappa \geq \aleph_{\omega}$.

Thus, we have demonstrated that Theorem 1 follows from Lemma 6. This proof actually delivers slightly more than is stated in Theorem 1.

Recall that the free $\boldsymbol{G L} \boldsymbol{L}$-algebra on a set of generators $V$ can be defined as the Lindenbaum algebra of GLB in the vocabulary $V$, that is, the modal boolean algebra of all formulas in the language of GLB (in variables $V$ ) modulo provable equivalence in GLB (see [8]). We have the following result, which is already of some interest when $V$ is countable or even finite.

Theorem $2\left(\square_{\aleph_{n}}\right.$, for $\left.n<\omega\right)$. Suppose $|V|<\aleph_{\omega}$. Then the free $\boldsymbol{G L} \boldsymbol{B}$-algebra on generators $V$ is embeddable into the algebra of the $\boldsymbol{G L} \boldsymbol{B}$-space $\left(\kappa ; \tau_{0}, \tau_{1}\right)$, for any $\kappa \geq \aleph_{\omega}$.

Proof. We only sketch an easy proof. Let $\lambda=\max \left(\aleph_{0},|V|\right)$. Enumerate all finite $J$-models in the vocabulary $V$ of $R_{1}$-height $n$ in a sequence of type $\lambda$ and denote their disjoint union $\mathcal{W}_{n}$. As above, construct a surjective weak $d$-map

$$
f_{n}:\left[1, \kappa_{n}\right] \rightarrow \mathcal{W}_{n},
$$

where $\kappa_{n} \leq \aleph_{n+1} \cdot \lambda$ (ordinal multiplication). Then, using Lemma 9, join all these maps $f_{n}$ for all $n<\omega$ into a surjective weak $d$-map $f:\left[1, \aleph_{\omega}\right) \rightarrow \mathcal{W}$, where $\mathcal{W}$ is the union of all finite $J$-models in the vocabulary $V$. To define the embedding of algebras, map any formula $\varphi$ to the set $\left\{\alpha \in\left[1, \aleph_{\omega}\right): \mathcal{W}, f(\alpha) \vDash \varphi\right\}$. The analog of Lemma 7 shows that this is, indeed, an embedding.

Notice that this theorem is an analog of the so-called uniform Solovay theorem in provability logic (see [2]). The rest of the paper is devoted to the proof of Lemma 6.

\section{Proof of Embedding Lemma}

We argue by induction on the $R_{0}$-height of $\mathcal{W}$.

BASIS. If $R_{0}$-height of $\mathcal{W}$ is 0 then $\mathcal{W}$ consists of a single 1 -sheet ordered by $R_{1}$ with a root $r$. By Lemma 2 there is a $\kappa$ and a surjective $d$-map $\left([1, \kappa], \tau_{1}\right) \rightarrow \mathcal{W}$. 
For the associated map $S$ conditions 1, 2, 4 hold by definition of $d$-map. Condition 3 holds trivially (its premise is always false). Condition 5 holds, because $S_{r}=\{\kappa\}$ and hence

$$
\bigcup_{y \in \widetilde{R}_{0}(x)} S_{y}=\bigcup_{y \in R_{1}(r)} S_{y}=[1, \kappa),
$$

which contains an end-segment of $\kappa$ and of any limit $\lambda<\kappa$.

InduCtion STEP. Since $\mathcal{W}$ is tree-like, it consists of the root 1 -sheet $\mathcal{W}_{0}$ (with its $R_{1}$-root $r_{0}$ ) and of the immediate subframes $\mathcal{W}_{1}, \ldots, \mathcal{W}_{n}$. By the induction hypothesis we have embeddings $S^{i}$ of $\mathcal{W}_{i}$ to $\left[1, \kappa_{i}\right]$, for $i=1, \ldots, n$. By Lemma 2 we also obtain a $d$-map $f_{0}:\left(\left[1, \kappa_{0}\right] \cap \operatorname{Lim}, \tau_{1}\right) \rightarrow \mathcal{W}_{0}$ and the associated embedding $S^{0}$. We set:

$$
\kappa:=\left(\kappa_{1}+\cdots+\kappa_{n}\right) \cdot \kappa_{0} .
$$

Define a partition $\left\{S_{x}: x \in \mathcal{W}\right\}$ as follows:

$$
\begin{aligned}
S_{x}:= & \left\{\left(\kappa_{1}+\cdots+\kappa_{n}\right) \cdot \alpha: \alpha \in S_{x}^{0}\right\}, \quad \text { if } x \in \mathcal{W}_{0} ; \\
S_{x}:= & \left\{\left(\kappa_{1}+\cdots+\kappa_{n}\right) \cdot \alpha+\kappa_{1}+\cdots+\kappa_{i}+\beta: \alpha<\kappa_{0}, \beta \in S_{x}^{i+1}\right\}, \\
& \quad \text { if } x \in \mathcal{W}_{i+1} \text { and } 0 \leq i<n .
\end{aligned}
$$

Denote:

$$
\begin{aligned}
\delta & :=\kappa_{1}+\cdots+\kappa_{n}, \\
\delta_{i} & :=\kappa_{1}+\cdots+\kappa_{i} .
\end{aligned}
$$

Obviously, sets of the form (1) partition the set $\left\{\delta \alpha: \alpha \in \operatorname{Lim} \cap\left[1, \kappa_{0}\right]\right\}$, moreover $S_{r_{0}}=\{\kappa\}$. Sets of the form (2) partition

$$
\left\{\delta \alpha+\delta_{i}+\beta: \alpha<\kappa_{0}, \beta \in\left[1, \kappa_{i+1}\right]\right\} .
$$

For different $i$ these sets do not intersect and their union is

$$
\left\{\delta \alpha+\delta: \alpha<\kappa_{0}\right\}=\left\{\delta \alpha: \alpha \in \operatorname{Suc} \cap\left[1, \kappa_{0}\right]\right\} .
$$

This yields Condition 1.

Condition 2. Suppose $\lambda \in S_{x}, x R_{1} y$. If $x \in \mathcal{W}_{i+1}$ then $\lambda=\delta \alpha+\delta_{i}+\lambda^{\prime}$, where $\lambda^{\prime} \in S_{x}^{i+1}$. Since $S_{y}^{i+1}$ is stationary in $\lambda^{\prime}$ the set $\left\{\delta \alpha+\delta_{i}+\beta: \beta \in S_{y}^{i+1}\right\}$ is stationary in $\lambda$. Then $S_{y}$ is also stationary in $\lambda$. For the same reason $\operatorname{cf}(\lambda)=$ $\operatorname{cf}\left(\lambda^{\prime}\right)>\omega$.

If $x \in \mathcal{W}_{0}$ then $\lambda=\delta \lambda^{\prime}$ with $\lambda^{\prime} \in S_{x}^{0}$. We claim that $\left\{\delta \alpha: \alpha \in S_{y}^{0}\right\}$ is stationary in $\lambda$.

Consider any club $C$ in $\lambda$. Since $D:=\left\{\delta \alpha: \alpha<\lambda^{\prime}\right\}$ is a club in $\lambda, C \cup D$ is also a club. Hence, $C_{1}:=\{\alpha: \delta \alpha \in C\}$ is a club in $\lambda^{\prime}$. Therefore, $C_{1} \cap S_{y}^{0} \neq \varnothing$, so there is an $\alpha \in S_{y}^{0}$ such that $\delta \alpha \in C$. 
Condition 3. Suppose $\lambda \in S_{x}, x R_{0} y$. If $x \in \mathcal{W}_{i+1}$ then $\lambda=\delta \alpha+\delta_{i}+\lambda^{\prime}$, where $\lambda^{\prime} \in S_{x}^{i+1}$ and $y \in \mathcal{W}_{i+1}$. Then, clearly $\left\{\delta \alpha+\delta_{i}+\beta: \beta \in S_{y}^{i+1}\right\}$ is unbounded in $\lambda$.

If $x \in \mathcal{W}_{0}$ and $y \in \mathcal{W}_{i+1}$, then $\lambda=\delta \lambda^{\prime}$ with $\lambda^{\prime} \in S_{x}^{0}$, and therefore $\lambda^{\prime} \in$ Lim. Select a $\beta_{0} \in S_{y}^{i+1}$. The set $\left\{\delta \alpha+\delta_{i}+\beta_{0}: \alpha<\lambda^{\prime}\right\}$ is unbounded in $\lambda$ and is contained in $S_{y}$.

Condition 4. Suppose $\lambda \in S_{x}, \operatorname{cf}(\lambda)>\omega$. If $x \in \mathcal{W}_{i+1}$ then $\lambda=\delta \alpha+\delta_{i}+\lambda^{\prime}$, where $\lambda^{\prime} \in S_{x}^{i+1}$. The set $\bigcup_{y \in R_{1}(x)} S_{y}^{i+1}$ contains a club in $\lambda^{\prime}$. Then

$$
\left\{\delta \alpha+\delta_{i}+\beta: \beta \in \bigcup_{y \in R_{1}(x)} S_{y}^{i+1}\right\}
$$

contains a club in $\lambda$. This set equals

$$
\bigcup_{y \in R_{1}(x)}\left\{\delta \alpha+\delta_{i}+\beta: \beta \in S_{y}^{i+1}\right\},
$$

which is contained in $\bigcup_{y \in R_{1}(x)} S_{y}$.

If $x \in \mathcal{W}_{0}$ then $\lambda=\delta \lambda^{\prime}$ with $\lambda^{\prime} \in S_{x}^{0}$. The set $\bigcup_{y \in R_{1}(x)} S_{y}^{0}$ contains a club in $\lambda^{\prime}$. Then $\left\{\delta \beta: \beta \in \bigcup_{y \in R_{1}(x)} S_{y}^{0}\right\}$ contains a club in $\lambda$. But this set equals

$$
\bigcup_{y \in R_{1}(x)}\left\{\delta \beta: \beta \in S_{y}^{0}\right\}=\bigcup_{y \in R_{1}(x)} S_{y} .
$$

Condition 5. Suppose $\lambda \in S_{x}, \lambda \in \operatorname{Lim}$. If $x \in \mathcal{W}_{0}$ then $\lambda=\delta \lambda^{\prime}$ with $\lambda^{\prime} \in S_{x}^{0}$. Since $\widetilde{R_{0}}(x)=\mathcal{W}-\left\{r_{0}\right\}$ we have

$$
\bigcup_{y \in \widetilde{R_{0}}(x)} S_{y}=[1, \kappa) .
$$

Since $\lambda \leq \kappa$ the set $\bigcup_{y \in \widetilde{R_{0}}(x)} S_{y}$ contains an end-segment of $\lambda$.

If $x \in \mathcal{W}_{i+1}$ then $\lambda=\delta \alpha+\delta_{i}+\lambda^{\prime}$, where $\lambda^{\prime} \in S_{x}^{i+1}$. We have that $\bigcup_{y \in \widetilde{R_{0}}(x)} S_{y}^{i+1}$ contains an end-segment of $\lambda^{\prime}$ and $\widetilde{R_{0}}(x) \subseteq \mathcal{W}_{i+1}$. Then

$$
\left\{\delta \alpha+\delta_{i}+\beta: \beta \in \bigcup_{y \in \widetilde{R}_{0}(x)} S_{y}^{i+1}\right\}
$$

contains an end-segment of $\lambda$. But this set is contained in $\bigcup_{y \in \widetilde{R_{0}}(x)} S_{y}$.

\section{Concluding remarks}

We have shown that the completeness of GLB is consistent with the axioms of ZFC (provided ZFC itself is consistent). The fact that the incompleteness of 
GLB is consistent with ZFC directly follows from the results of Blass (which, in turn, rely on some deep results of Harrington and Shelah concerning stationary reflection). We do not have to go deeply into set theory here, as it is well-known that GLB is conservative over the axioms of GL for the language restricted to any individual modality. Hence, the completeness of GLB implies the completeness of GL under the interpretation w.r.t. the club topology. Assuming that there are models of ZFC with Mahlo cardinals, Blass has shown that there is a model of ZFC in which GL is incomplete w.r.t. the club topology. Hence, the same model demonstrates the incompleteness of GLB w.r.t. the considered interpretation.

In the other direction, following [11, Theorem 6], we remark that by a result of Jensen [21] the negation of $\square_{\kappa}$ implies that $\kappa^{+}$is Mahlo in $L$. Hence, by Theorem 1, if GLB is incomplete there are Mahlo cardinals in $L$. Thus, we obtain the second part of the following corollary.

Corollary 3. (i) The statement that $\boldsymbol{G} \boldsymbol{L} \boldsymbol{B}$ is complete w.r.t. the class of spaces of the form $\left(\kappa ; \tau_{0}, \tau_{1}\right)$ is equiconsistent with ZFC;

(ii) The statement that $\boldsymbol{G} \boldsymbol{L} \boldsymbol{B}$ is incomplete w.r.t. the class of spaces of the form $\left(\kappa ; \tau_{0}, \tau_{1}\right)$ is equiconsistent with the existence of Mahlo cardinals.

\section{References}

1. M. Abashidze. Ordinal completeness of the Gödel-Löb modal system (Russian). In Intensional logics and the logical structure of theories, pages 49-73. Metsniereba, Tbilisi, 1985.

2. S.N. Artemov and L.D. Beklemishev. Provability logic. In D. Gabbay and F. Guenthner, editors, Handbook of Philosophical Logic, 2nd ed., volume 13, pages 229-403. Kluwer, Dordrecht, 2004.

3. L.D. Beklemishev. Provability algebras and proof-theoretic ordinals, I. Annals of Pure and Applied Logic, 128:103-123, 2004.

4. L.D. Beklemishev. Reflection principles and provability algebras in formal arithmetic. Uspekhi Matematicheskikh Nauk, 60(2):3-78, 2005. In Russian. English translation in: Russian Mathematical Surveys, 60(2): 197-268, 2005.

5. L.D. Beklemishev. The Worm Principle. In Z. Chatzidakis, P. Koepke, and W. Pohlers, editors, Lecture Notes in Logic 27. Logic Colloquium '02, pages 75-95. AK Peters, 2006. Preprint: Logic Group Preprint Series 219, Utrecht University, March 2003.

6. L.D. Beklemishev. Kripke semantics for provability logic GLP. Annals of Pure and Applied Logic, 161:756-774, 2010. Preprint: Logic Group Preprint Series 260, University of Utrecht, November 2007. http://preprints.phil.uu.nl/lgps/.

7. L.D. Beklemishev. On the Craig interpolation and the fixed point property for GLP. Logic Group Preprint Series 262, University of Utrecht, December 2007. http://preprints.phil.uu.nl/lgps/.

8. L.D. Beklemishev, G. Bezhanishvili, and T. Icard. On topological models of GLP. Logic Group Preprint Series 278, University of Utrecht, August 2009. http://preprints.phil.uu.nl/lgps/.

9. L.D. Beklemishev, J. Joosten, and M. Vervoort. A finitary treatment of the closed fragment of Japaridze's provability logic. Journal of Logic and Computation, 15(4):447-463, 2005. 
10. G. Bezhanishvili, L. Esakia, and D. Gabelaia. Some results on modal axiomatization and definability for topological spaces. Studia Logica, 81:325-355, 2005.

11. A. Blass. Infinitary combinatorics and modal logic. Journal of Symbolic Logic, 55(2):761-778, 1990.

12. G. Boolos. The analytical completeness of Dzhaparidze's polymodal logics. Annals of Pure and Applied Logic, 61:95-111, 1993.

13. G. Boolos. The Logic of Provability. Cambridge University Press, Cambridge, 1993.

14. L. Carlucci. Worms, gaps and hydras. Mathematical Logic Quarterly, 51(4):342$350,2005$.

15. L. Esakia. Diagonal constructions, Löb's formula and Cantor's scattered space (Russian). In Studies in logic and semantics, pages 128-143. Metsniereba, Tbilisi, 1981.

16. L. Esakia. Intuitionistic logic and modality via topology. Annals of Pure and Applied Logic, 127:155-170, 2003.

17. T.F. Icard, III. A topological study of the closed fragment of GLP. Journal of Logic and Computation, 2009. Advance Access published online on August 12, 2009, doi: 10.1093/logcom/exp043.

18. K.N. Ignatiev. On strong provability predicates and the associated modal logics. The Journal of Symbolic Logic, 58:249-290, 1993.

19. G.K. Japaridze. The modal logical means of investigation of provability. Thesis in Philosophy, in Russian, Moscow, 1986.

20. T. Jech. Set Theory. The Third Millenium Edition. Springer, 2002.

21. R.B. Jensen. The fine structure of the constructible hierarchy. Annals of Mathematical Logic, 4:229-308, 1972.

22. J. C. C. McKinsey and A. Tarski. The algebra of topology. Annals of Mathematics, 45:141-191, 1944. 